\title{
Interaural time difference sensitivity under binaural cochlear implant stimulation even at high pulse rates up to 900 pps
}

Alexa N. Buck ${ }^{1,3 \# ;}$; Sarah Buchholz ${ }^{2}$ Jan W. Schnupp ${ }^{1,3}$; Nicole Rosskothen-Kuhl ${ }^{1,2^{*} \#}$

1) Department of Neuroscience, City University of Hong Kong, Hong Kong (SAR China);

2) Department of Oto-Rhino-Laryngology, Section of Experimental and Clinical Otology, Medical Center - University of Freiburg, Faculty of Medicine, University of Freiburg,

Germany

3) City University of Hong Kong Shenzhen Research Institute, Shenzhen, China.

\#These authors contributed equally to this work.

\section{Contact Info}

$15{ }^{*}$ Corresponding author:

Dr. Nicole Rosskothen-Kuhl

Neurobiological Research Laboratory,

Section for Experimental and Clinical Otology,

Medical Center - University of Freiburg,

20 Killianst. 5,

79106 Freiburg i. Br., Germany

Phone: +49 76127042730

E-mail: nicole.rosskothen-kuhl@uniklinik-freiburg.de

\section{Keywords}

Deafness; prosthetics; cochlear implant; binaural hearing; interaural time difference; psychoacoustics; hearing experience 


\section{Abstract}

30 Spatial hearing remains one of the major challenges for bilateral cochlear implant (biCl) users, and early deaf patients in particular are often completely insensitive to interaural time differences (ITDs) delivered through biCls. One popular theory held is that this may be due to a lack of early binaural experience. However, previously we have shown that neonatally deafened rats fitted with biCls in adulthood quickly learn to discriminate ITDs as

35 well as their normal hearing litter mates (Rosskothen-Kuhl et al. 2021), and perform orders of magnitude better than human biCl users. Our unique behaving biCl rat model allows us to investigate other possible limiting factors of prosthetic binaural hearing, such as the effect of stimulus pulse rate and envelope shape. Previous work has indicated that ITD sensitivity may decline substantially at the high pulse rates often used in clinical practice.

40 We therefore measured behavioral ITD thresholds in neonatally deafened, adult implanted biCl rats to pulse trains of 50,300, 900 and 1800 pulses per second (pps), with either rectangular or Hanning window envelopes. Our rats exhibited very high sensitivity to ITDs at pulse rates up to 900 pps for both envelope shapes, a pulse rate commonly used in clinical practice. However, ITD sensitivity declined to near zero at 1800 pps, for both

45 Hanning and rectangular windowed pulse trains. Current clinical cochlear implant $(\mathrm{Cl})$ processors are often set to pulse rates $\geq 900$ pps, but ITD sensitivity in human CI listeners is often reported to decline sharply above $\sim 300 \mathrm{pps}$. Our results suggest that the relatively poor ITD sensitivity seen at $>300$ pps in human $\mathrm{Cl}$ users may not reflect the hard upper limit of biCI ITD performance in the mammalian auditory pathway. Perhaps with training or

50 better $\mathrm{Cl}$ strategies good binaural hearing may be achievable at pulse rates high enough to allow good sampling of speech envelopes while delivering usable ITDs.

\section{Introduction}

To date, cochlear implants (Cls) have been provided to over 500,000 severely hearing

55 impaired patients across the globe (Ear Foundation, UK, 2016) and have greatly improved the quality of life of their recipients. However, substantial limitations remain in the $\mathrm{Cl}$ hearing experience. For example, $\mathrm{Cl}$ users suffering from prelingual deafness show invariably poor interaural time difference (ITD) performances, with even rare star performers only achieving thresholds of a few hundred $\mu$ s (Poon et al. 2009; Conti-

60 Ramsden et al. 2012; Litovsky et al. 2012; Goupell and Litovsky 2014; Laback et al. 2015; Litovsky and Gordon 2016; Ehlers et al. 2017; Thakkar et al. 2020). This poor ITD sensitivity is often hypothesized to be a result of the absence of auditory experience during 
an early critical period (Kral 2013). However, in Rosskothen-Kuhl et al. (2021) we used a rat model to show that, even in the absence of early auditory input, near normal ITD

65 sensitivities can be obtained, at least for low pulse rates, when stimuli were provided with precisely synchronized $\mathrm{Cl}$ processors. This indicates that developmental critical periods may not be the main reason for the poor ITD sensitivity seen in bilateral $\mathrm{Cl}$ (biCl) users, and that technological shortcomings may instead be the main limiting factors.

One critical factor is likely to be the pulse rate at which Cls operate. For example, a review

70 by Laback et al. (2015) concludes that ITD performance of postlingually deaf biCl users tends to decline as pulse rates increase above $\sim 300$ pulses per second (pps). Several previous studies on human listeners (van Hoesel and Tyler 2003; van Hoesel 2004; Majdak et al. 2006; van Hoesel 2007; Laback et al. 2007; van Hoesel 2008) as well as physiological measures of sensitivity to ITDs obtained from experimental animals (Smith

75 and Delgutte 2007; Chung et al. 2016; Chung et al. 2019) generally support this conclusion. However, accurately characterizing the dependence of ITD sensitivity on pulse rates in human listeners is marred with difficulties. The wide variety of patient histories and the very limited control that researchers have over variables such as the patients' $\mathrm{Cl}$ hardware, implantation history, stimulation parameters, experience, daily routine or

80 availability for psychoacoustic testing introduce numerous confounds, which make it very difficult to isolate the effect of pulse rate and to quantify it with high accuracy under otherwise favorable conditions. This context makes the recent development of a behavioral biCl rat model (Rosskothen-Kuhl et al. 2021) invaluable. Normal hearing rats exhibit behavioral high frequency ("envelope") ITD thresholds which are very similar to

85 those seen in humans (Li et al. 2019), and even show very similar "precedence effects" as are seen in human ITD perception (Brown and Stecker 2010; Li et al. 2021). This makes rats a well validated model for human binaural hearing, well suited for characterizing the stimulus parameter ranges that allow high levels of ITD sensitivity with high accuracy and reproducibility.

90 Current $\mathrm{Cl}$ processors generally run at fixed pulse rates between 900 and 3700 pps (Middlebrooks 2008). The rationale here is that faster temporal sampling of speech envelopes might improve speech recognition in Cl users. However, Shannon et al. (2011) demonstrated that increasing pulse rates from 600 to 2400 pps resulted in little to no benefit for phoneme, word, and sentence recognition in quiet or in noise.

95 Generally speaking, modern $\mathrm{Cl}$ processor designers face conflicting demands: they must use pulse rates that are fast enough to sample the envelopes of speech and other sound 
inputs of interest with good detail, yet slow enough to allow ITD perception. However, neither the lower limits of pulse rates required for good speech encoding nor the upper limits of pulse rates for good ITD encoding have been determined definitively or with great accuracy, making it difficult to decide what pulse rates might be optimal for future biCl stimulation strategies. To start filling these important gaps in our knowledge, we conducted a series of positively reinforced two-alternative forced choice (2-AFC) ITD lateralization experiments on neonatally deafened (ND) rats which were fitted with biCls in early adulthood, measuring their behavioral ITD thresholds with binaural pulse trains at a variety

105 of pulse rates, and with either sharp (rectangular) or gentle (Hanning windowed) onsets and offsets. Based on previous studies in Cl listeners (Litovsky et al. 2012; Ehlers et al. 2017; Thakkar et al. 2020) and electrophysiology studies in Cl animals (Tillein et al. 2009; Hancock et al. 2012; Hancock et al. 2013; Chung et al. 2014; Chung et al. 2016; Tillein et al. 2016) we expected to see generally better ITD sensitivities for lower pulse rates and a 110 rapid drop off above 300 pps. In addition, we expected to observe reduced ITD lateralization performances when presenting pulse trains with gently rising slopes (Hanning windowed) rather than sharp onset rectangle windowed pulse trains considering previous physiological and behavioral studies on ITDs demonstrating the precedence effect for these binaural spatial cues (Brown and Stecker 2010; Li et al. 2021). This study demonstrates these expectations were in part born out in the data, but not nearly as strongly as one might suspect. In particular, the behavioral ITD sensitivity of our biCl rats often remained remarkably good even for clinically relevant pulse rates as high as 900 pps, and using Hanning as opposed to rectangular envelopes led to only modest reductions in ITD sensitivity.

\section{Results}

Sixteen ND rats received chronic biCls in early adulthood (postnatal weeks 10-14). All rats consistently showed well differentiated, electrically evoked auditory brainstem responses (eABRs) to intracochlear stimulation (Fig. S1). eABR recordings were repeated frequently

125 throughout the training period. All ND rats were successfully trained to localize ITD cues within 3-5 days of behavioral training (8 sessions on average with 2 sessions per day), regardless of the starting pulse rate (50, 300 or 900 pps). Figure 1 shows the experimental timeline and the number of $\mathrm{Cl}$ animals tested per condition. For details on the behavioral training and test setup see Rosskothen-Kuhl et al. (2021). Representative psychometric curves for ITD lateralization with rectangular windowed pulse trains from four of these ND 
$\mathrm{Cl}$ rats are shown in Figure 2. Supplementary Figure S2 shows the corresponding psychometric curves for all 16 animals tested for rectangle windowed stimuli. Uniformly good ITD sensitivity, as evidenced by steep slopes in the psychometric functions and very low error rates for absolute ITDs $\geq 80 \mu \mathrm{s}$, is seen at all pulse rates tested with the exception of 1800 pps. At 1800 pps the animals' performance dropped to near-chance with high variability in performance, as seen by the large error bars (Figs. 2, S3) even at large ITDs; whereas at lower pulse rates the same animals were performing well over $80 \%$ correct for ITDs $\geq 60 \mu \mathrm{s}$. In two of the $\mathrm{Cl}$ animals shown in Figure 2, the decrease in ITD performance was accompanied by a bias for the rat's left spout (NDCl-14 and NDCl-15).

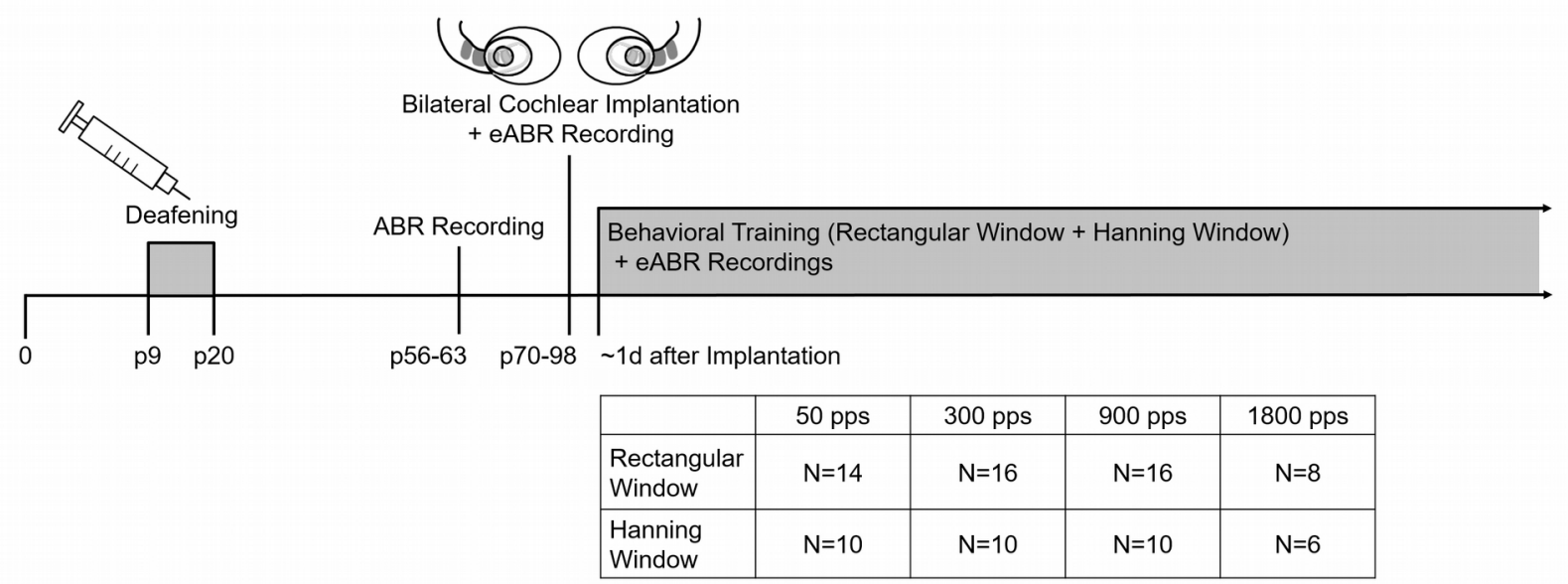

Figure 1: Experimental pipeline with timeline and number of animals $(\mathrm{N})$ tested per condition. Abbreviations: ABR = acoustically evoked auditory brainstem response, eABR $=$ electrically evoked auditory brainstem response, $p=$ postnatal day, $p p s=$ pulses per second, $d=$ day. 

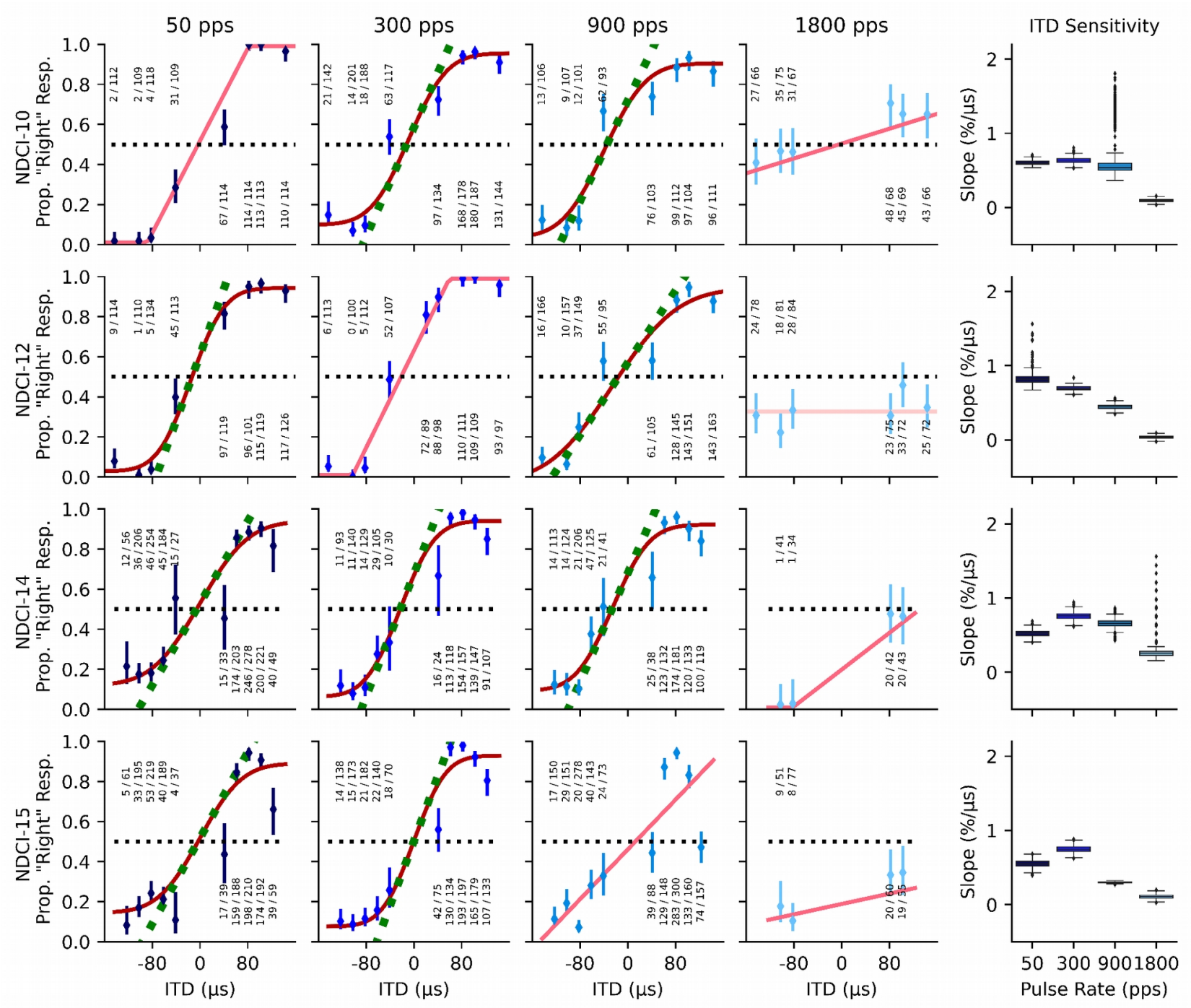

Figure 2: Psychometric curves for four example neonatally deafened, cochlear implanted $(\mathrm{NDCl})$ rats localizing rectangular windowed pulse trains by interaural time difference (ITD). The first four columns represent a different pulse rate from left to right $50,300,900$, and 1800 pulses per second (pps), each indicated with a different shade of blue. The $y$-coordinates reflect the proportion of trials during which the animal responded on the "right" hand spout (Prop. "Right" Resp.). The x-axis shows the tested ITD values from -150 to $+150 \mu \mathrm{s}$. Negative ITD values indicate left leading ITDs. Annotations above or below each marker indicate the number of trials the animal chose the right hand side spout over the total number of presentations for the given ITD value on the abscissa. From dark to light the different shades of red indicate sigmoid, linear with bounds or null model psychometric curve fits. Green dashed lines show slopes of psychometric curves at ITD $=0$. Slopes serve to quantify the behavioral sensitivity of the animal to ITDs. The fifth column summarizes the ITD sensitivity across the different pps per animal as a function of slope. Each row shows the responses for a given animal. Psychometrics of all $\mathrm{Cl}$ animals are shown in Figure S2.

In addition 10 out of 16 animals were tested for ITD sensitivity to pulse trains which were 145 amplitude modulated with a slow rising and falling Hanning window at 50, 300 and 900 pps $(\mathrm{N}=10)$ as well as at 1800 pps $(\mathrm{N}=6)$ (see Figs. 1 and $\mathrm{S} 3$ for details). Again, all animals 
showed good ITD sensitivity with steep slopes in the psychometric curves from the 2-AFC task at all pulse rates except for 1800 pps (Fig. S3). At this pulse rate the psychometric functions were completely flat for 5 out of 6 animals tested as shown in the fourth column of Figures 3 and S3. The slopes of the psychometric functions for Hanning windowed pulse trains were generally found to be more shallow compared to those from the sharp onset rectangular windowed stimuli shown in Figures 2 and S2. In addition, more variability in the animals' ITD performances appeared under Hanning windowed stimuli (see Figs. 3, S3) compared to that under rectangle windowed stimuli across pulse rates (Figs. 2, S2).
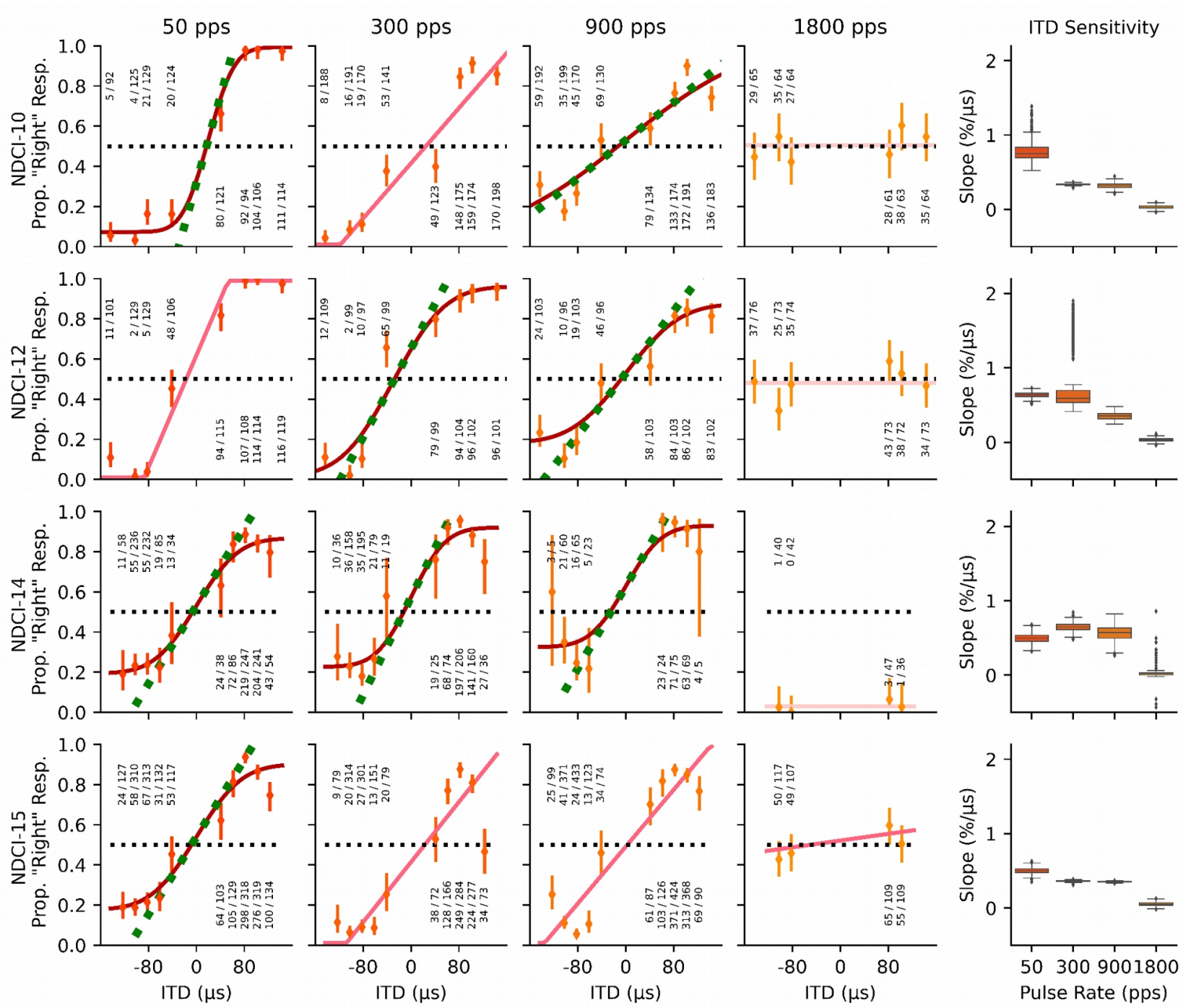

Figure 3: Psychometric curves for the same four example neonatally deafened, cochlear implanted $(\mathrm{NDCl})$ rats as shown in Figure 2 localizing Hanning windowed pulse trains with varying interaural time differences (ITDs). Details are as for Figure 2. Psychometrics of all $\mathrm{Cl}$ animals tested on Hanning windowed stimuli are shown in Figure S3.

To quantify the effect of pulse rate and pulse train envelope on ITD discrimination performance in $\mathrm{biCl}$ animals, we quantified behavioral sensitivity by the slope of the psychometric curve around the ITD $=0 \mu$ s midline. We then used bootstrap tests (see 
Methods for details) to estimate the distributions and confidence limits of these sensitivity values for all animals at each pulse rate and envelope type, and to estimate the probability that the observed differences in mean ITD sensitivity across our animals as a function of either pulse rate or envelope type were larger than expected by chance. Figure 4 shows the bootstrapped mean distributions of ITD sensitivities as a function of stimulus window type and pulse rate, averaged over all animals for which behavioral data at the relevant combination of stimulus parameters was available. The p-values for the null hypothesis that the sensitivity to ITD did not differ for a given pair of stimulus parameter combinations were Bonferroni corrected to account for multiple comparisons and are listed in Tables 1, 2 and 3. ITD sensitivities were usually higher with rectangular than Hanning windowed envelopes, and that effect was statistically significant at 300 and 900 pps. ITD sensitivity 175 also tended to decline, often significantly, with increasing pulse rate, but good ITD sensitivity even with Hanning windowed stimuli was nevertheless common at pulse rates as high as 900 pps. In contrast, at 1800 pps, ITD sensitivity was generally poor independent of the presented window type.

Psychometric slopes are not necessarily a very intuitive metric for sensitivity. To help 180 readers put the observed slope values into perspective and compare them against ITD discrimination thresholds reported in other previous studies, we also show what sort of $75 \%$ correct discrimination thresholds one might expect from the animals given their psychometric slopes. These estimated 75\% ITD discrimination thresholds, in $\mu \mathrm{s}$, are shown on the right $y$-axis of Figure 4. Some authors, including Ulrich and Vorberg (2009) 185 and Lapid et al. (2008), refer to such 75\% discrimination thresholds as the "just-noticable difference" (JND). Note, however, that our animals were only tested on a lateralization task, not a discrimination task. Task details can influence performance, so readers may wish to use some discretion when comparing our JND scores against those reported elsewhere. Table 4 gives the mean $75 \%$ discrimination thresholds, or JNDs, as estimated

190 from the mean slopes of the psychometric curves (see Methods), for each of the eight different stimulation conditions. Overall, the smallest $75 \%$ discrimination thresholds were obtained for pulse rates of 50 and 300 pps with rectangular stimuli often resulting in better estimated discrimination. The only exception can be observed at 50 pps where Hanning windowed stimuli resulted in better discrimination thresholds than for rectangular windowed stimuli. For higher pulse rates, the estimated $75 \%$ discrimination threshold rose, but even at a clinical pulse rate of 900 pps, estimated JNDs were in the order of $50.5-62$ $\mu$ s which are well below ITD discrimination thresholds observed for most of the early deafened biCl patients which range from $200 \mu$ s to well above $1000 \mu s$ (van Hoesel and 
Clark 1997; van Hoesel et al. 2002; van Hoesel et al. 2009; Litovsky et al. 2012; Litovsky et al. 2013; Laback et al. 2015; Ehlers et al. 2017; Thakkar et al. 2020) Note that the values shown in Table 4 for 1800 pps represent gross extrapolations of very small and quite variable psychometric slopes well beyond the range of ITDs tested. Thus, while the mean threshold values shown for 50,300, and 900 pps can be considered reliable and accurate having been taken from the measured data, those at 1800 should be treated as very rough estimates.

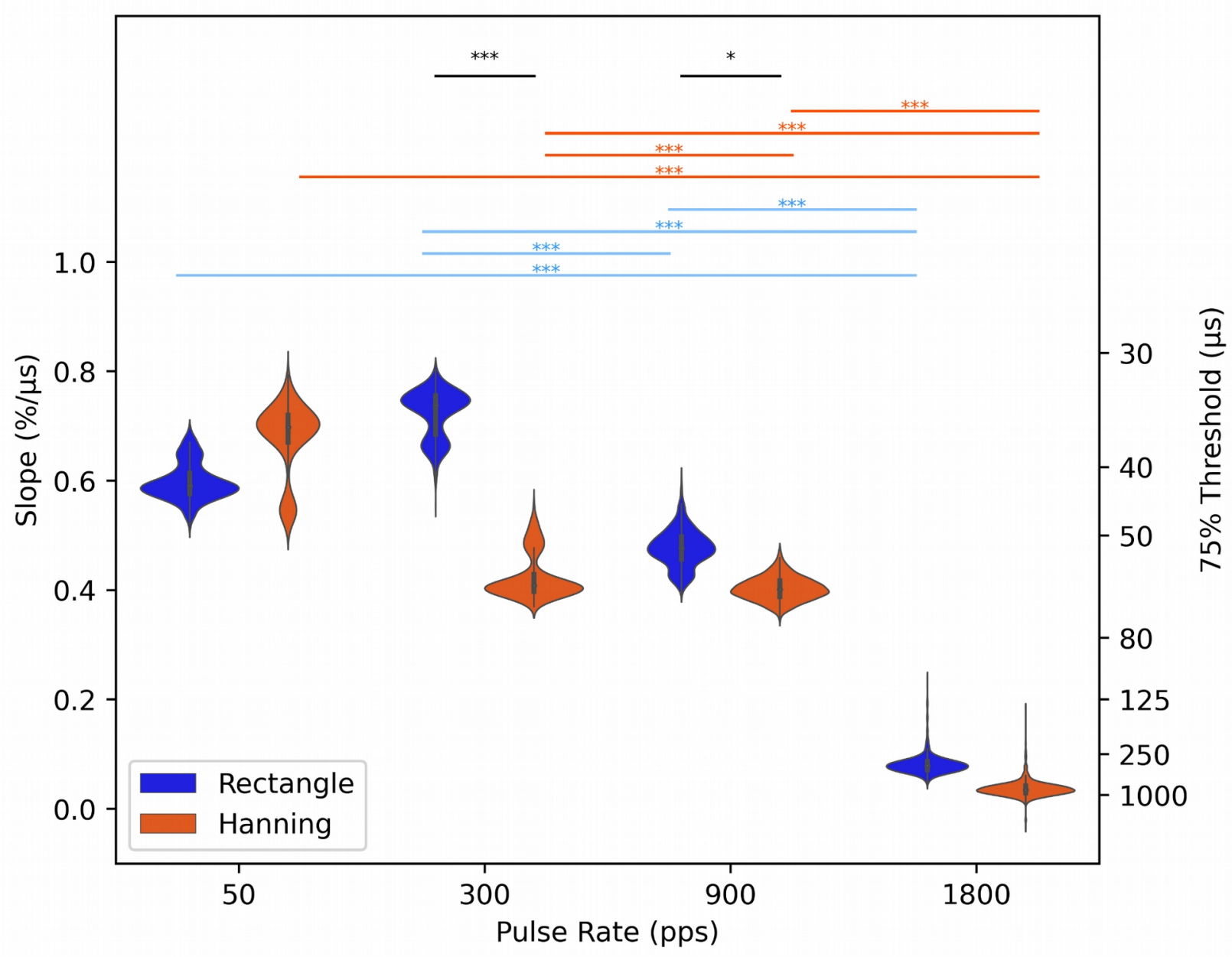

Figure 4: Violin plots showing the distribution of the mean group effects of ITD sensitivity for each pulse rate following permutation for rectangular (blue) and Hanning (orange) windowed stimuli. The left axis shows the ITD sensitivity as a function of the psychometric slopes, and the right $y$-axis the corresponding 75\% ITD discrimination threshold estimate. Significant group statistics for the slopes comparing pulse rates are shown above violin plots for rectangular (blue) and Hanning (orange) windowed data. Significant differences between the two envelopes are shown in black. ${ }^{* *}=(p<0.001),{ }^{* *}=(0.001 \leq p<0.01),{ }^{*}=$ $(0.01 \leq p<0.05)$. 


\begin{tabular}{|l|l|l|l|}
\hline Rectangle & 50 pps & 300 pps & 900 pps \\
\hline 300 pps & 0.491 & & \\
\hline 900 pps & 0.079 & $<0.001$ & \\
\hline 1800 pps & $<0.001$ & $<0.001$ & $<0.001$ \\
\hline
\end{tabular}

Table 1: Bonferroni corrected $p$-values for the null hypothesis that mean ITD sensitivities do not vary as a function of pulse rate for rectangular windowed stimuli with pps values shown in the row and column headings. Significant p-values $(<0.05)$ are shown in bold.

\begin{tabular}{|l|l|l|l|}
\hline Hanning & 50 pps & 300 pps & 900 pps \\
\hline 300 pps & 0.014 & & \\
\hline 900 pps & $<0.001$ & 1 & \\
\hline 1800 pps & $<0.001$ & $<0.001$ & $<0.001$ \\
\hline
\end{tabular}

Table 2: $p$-values as in Table 1, but for Hanning windowed stimuli.

\begin{tabular}{|l|l|l|l|l|}
\hline & at $50 \mathrm{pps}$ & at $300 \mathrm{pps}$ & at $900 \mathrm{pps}$ & at $1800 \mathrm{pps}$ \\
\hline $\begin{array}{l}\text { Hanning vs. } \\
\text { Rectangle }\end{array}$ & $>0.999$ & $<0.001$ & 0.022 & 0.061 \\
\hline
\end{tabular}

215 Table 3: Bonferroni corrected p-values for the null hypothesis that mean ITD sensitivities do not vary as a function of envelope shape, rectangular or Hanning windowed stimuli, when tested at the pps values shown in the column headings. Significant $p$-values $(<0.05)$ are shown in bold.

\begin{tabular}{|l|l|l|l|l|}
\hline $75 \%$ threshold & at $50 \mathrm{pps}$ & at $300 \mathrm{pps}$ & at $900 \mathrm{pps}$ & at $1800 \mathrm{pps}$ \\
\hline Rectangle & $43.2(\mathrm{~N}=14)$ & $34.6(\mathrm{~N}=16)$ & $50.5(\mathrm{~N}=16)$ & $401.5(\mathrm{~N}=8)$ \\
\hline Hanning & $40.5(\mathrm{~N}=10)$ & $61.8(\mathrm{~N}=10)$ & $62.0(\mathrm{~N}=10)$ & $3575.8(\mathrm{~N}=6)$ \\
\hline
\end{tabular}

220 Table 4: Mean estimated 75\% ITD discrimination thresholds in $\mu$, estimated from the slopes of the psychometric curves (see Methods) and averaged over all animals $(\mathrm{N})$ tested at the corresponding stimulus condition.

\section{Discussion}

\section{Early deafened biCl rats show good ITD lateralization for pulse rates up}

\section{5 to 900 pps independent of envelope shape}

In this study, we have demonstrated remarkably good ITD sensitivity despite early onset deafness under bilateral electric stimulation, including at electrical pulse rates in the range of those commonly used in current clinical practice. Even with Hanning windowed stimuli, all of our biCl rats routinely performed above $80 \%$ correct with ITDs greater than or equal 
to $80 \mu \mathrm{s}$ at pulse rates of 900 pps. This gives rise to the hope that it may in principle be possible to devise binaural $\mathrm{Cl}$ processing strategies capable of delivering very useful ITD cues to patients listening to speech enveloped stimuli. It should be emphasized that our animals received informative ITDs with microsecond precise relative timing of stimulus pulses delivered to each ear from the onset of their bilateral stimulation. Current clinical practice does not deliver binaural stimulation conditions, which are favorable for the development of good ITD sensitivity, to any human Cl listeners. Consequently it is perhaps not surprising that the good ITD sensitivity we observe in our rats contrasts with the results obtained from human biCl users. Prelingually deaf $\mathrm{Cl}$ users often fail to develop any usable sensitivity to ITDs (Litovsky 2010; Ehlers et al. 2017). Cl users who do exhibit at least some ITD sensitivity tend to show a rapid drop-off in ITD lateralization performance with pulse rates above 300-500 pps (Laback et al. 2015). We also observed that ITD sensitivity was significantly lower with slowly rising Hanning envelopes than with very fast onset rectangular windowed pulses at rates of 300 and 900 pps. This is in qualitative agreement with studies in human $\mathrm{Cl}$ listeners that have reported a reduced ability to use ITD cues in stimuli with speech waveforms (Laback et al. 2004; Grantham et al. 2008) 245 which also consist of slow rising envelopes. While there is evidence that $\mathrm{Cl}$ users may be able to use ITDs carried on slow rising envelopes, human studies demonstrated that this is only possible when the shape of the envelope is peaked (Laback et al. 2004; van Hoesel et al. 2009; Laback et al. 2011; Noel and Eddington 2013). This strongly suggests that a speech envelope, or any slow rising envelope for that matter (such as a Hanning

250 windowed pulse train), delivered by current clinical speech processors does not contain the temporal features needed for effective encoding of ITD information. In addition, better ITD discrimination in stimuli with rapid onsets is perhaps unsurprising given that, for normal hearing subjects, onset ITDs are normally the most salient (Brown and Stecker 2010). Electrophysiological studies of ITD sensitivity have similarly demonstrated the

255 importance of onset cues in ITD sensitivity at least at the level of the inferior colliculus (Buck et al. 2021; Haqqee et al. 2021; Li et al. 2021) with a fall in ITD sensitivity of between 24-48\% when onset responses were excluded (Buck et al. 2021). Comparable results were also observed in a behavioral study in normal hearing rats across different pulse rates (Li et al. 2019).

260 In addition, ITD sensitivity under biCl stimulation has been shown to decrease with increasing pulse rates in studies when the onset cues were both included (Chung et al. 2016) or excluded (Smith and Delgutte 2007; Hancock et al. 2012; Hancock et al. 2013; Chung et al. 2019). For example, Chung et al. (2016) identified the best ITD sensitivity in 
IC for pulse rates around 80-160 pps followed by a degraded sensitivity for higher pulse

265 rates. However, in contrast to lower pulse rates, which are expected to improve ITD sensitivity (Joris and Yin 1998; van Hoesel 2007; Laback et al. 2007; Chung et al. 2016), there is evidence that temporal encoding is only improved at higher electrical pulse rates (Vollmer et al. 1999; Sunwoo et al. 2021)which again is interesting based on the evidence that ITD processing and temporal acuity may have similar mechanics (Snyder et al. 1995;

270 Vollmer et al. 1999).

Our behavioural results in $\mathrm{biCl}$ animals presented here thus show similar trends to those seen in previous electrophysiological work, namely that ITD sensitivity may decline if stimulus envelopes are lacking pronounced onsets or if pulse rates are high (Hancock et al. 2012; Hancock et al. 2013; Chung et al. 2016; Buck et al. 2021). However, the most

275 important observation to take away from our study is that, as already mentioned, our rats routinely achieved ITD thresholds well below $100 \mu$ s at 900 pps even with slowly rising Hanning envelopes. Thus, neither high carrier rates nor slow rising envelopes preclude the ability of biCl users to make use of ITD cues. If similar sensitivity to this binaural cue could be achieved with patients with improved $\mathrm{Cl}$ techniques, this could potentially result in

280 significant improvements in ITD performances and subsequent improvements in speech reception thresholds in noise.

\section{The role of pulse rate and stimulation position in ITD perception of $\mathrm{Cl}$ users}

285 The ITD sensitivity of our $\mathrm{Cl}$ animals even at pulse rates as high as $900 \mathrm{pps}$ is surprisingly good when compared to the average ITD discrimination performance of postlingually deaf human biCl users, which show a drop in sensitivity for pulse rates above 300-500 pps (Majdak et al. 2006; van Hoesel 2007; Thakkar et al. 2018). There appears to be a general consensus that, for good ITD perception, pulse rates should ideally be quite low, but they

290 do of course need to be fast enough to enable the high effective encoding of speech. However, that constraint need not be a major problem, since studies by Shannon et al. (2011) and Green et al. (2012) demonstrate that increasing the pulse rates above 600, at least up to 2400 pps, resulted in little to no benefit for phoneme, word and sentence recognition either in quiet or in noise.

295 To overcome the limited ITD sensitivity in biCl listeners, new strategies have been tried in studies on humans (Eklöf and Tideholm 2018; Srinivasan et al. 2018; Thakkar et al. 2018) 
and animals (Buechel et al. 2018). For example, configurations with mixed stimulation rates, in which one low rate electrode was included among an array of higher rate electrodes, inducing ITD sensitivity similar to that seen with configurations comprising only low rates (Thakkar et al. 2018). In addition, stimulation strategies have been developed that allow the encoding of the fine structure of sound. In Eklöf and Tideholm (2018), 50\% of the listeners using such a fine structure strategy showed an ITD sensitivity with a mean threshold at $330 \mu \mathrm{s}$, while listeners without fine structure strategy failed to detect ITDs > $1000 \mu s$. Another proposed strategy involved combining both high and low pulse rates

305 together. Srinivasan et al. (2018) report that the introduction of an additional slower pulse train overlying a faster pulse train, but with a small offset, improves ITD sensitivity beyond what might be expected from a simple, equivalent increase in stimulus amplitude. In biCl rabbits, Buechel et al. (2018) have shown that adding extra pulses to periodic pulse trains of high pulse rates introduces short inter-pulse intervals resulting in better ITD coding of auditory midbrain neurons.

These new stimulation strategies are ingenious, but it is noteworthy that, even with the improvements that they may have induced, the ITD discrimination of the human $\mathrm{Cl}$ listeners tested still falls short of the performance that our early deafened rats were able to achieve at high pulse rates of up to 900 pps and without special strategies. This suggests

315 that the factors limiting ITD perception in human biCl users may originate from other factors. For example, the $\mathrm{Cl}$ stimulation our rats received always contained highly precise and informative ITD cues from the very beginning of implantation, where as the majority of binaural stimulation delivered to human $\mathrm{Cl}$ listeners comes from current standard issue clinical processors which deliver uninformative ITDs. Our work thus raises the possibility

320 that providing users with consistently precise and informative ITD cues might allow them to develop very good ITD sensitivity at clinically relevant pulse rates, at least up to 900 pps. Using lower pulse rates might improve ITD sensitivity further, as has been shown in previous studies on animal and human $\mathrm{Cl}$ users (Smith and Delgutte 2007; van Hoesel et al. 2009; Hancock et al. 2012; Kan and Litovsky 2015; Laback et al. 2015; Chung et al.

325 2016), and has again been demonstrated here, but there should be plenty of scope to deliver very useful ITD cue information at pulse rates well within the range needed for effective speech coding.

In addition to stimulation strategies with modified or mixed pulse rates, the effects of changing intracochlear stimulation positions on ITD sensitivity in biCl users have also been considered in several studies. Shannon et al. (2011) and Thakkar et al. (2018) reported 
that increasing the number of activated stimulation electrodes could improve both ITD localization and speech performance of $\mathrm{Cl}$ users. Whether this was due to the increased amplitude and energy spread, thus increasing the chance of stimulating surviving spiral ganglion cells, or a result of wider frequency activation, is still an open question. It is possible that wider activation increases the energy reaching the apex of the cochlea, and thus the low frequency region, which, according to the duplex theory (Schnupp et al. 2011), is often thought of as particularly attuned to ITD processing. Moreover, directly stimulating the low frequency pathways in an animal model with monaural penetrating auditory nerve electrodes results in better temporal acuity than that observed when higher

340 frequency fibres are stimulated with the same types of electrodes (Middlebrooks and Snyder 2007; Middlebrooks and Snyder 2010). Note that human intracochlear electrodes implanted with current tools and surgical techniques usually fail to reach the apical, most low-frequency end of the cochlea (Stakhovskaya et al. 2007). One might expect that this could reduce ITD sensitivity overall, but also that the best ITD sensitivity might be

345 observed if the apical-most electrodes are stimulated. However, Kan et al. (2015) observed that ITD sensitivity could be optimized with a stimulation of sets of electrodes that spanned the full length of the $\mathrm{Cl}$ electrode array. It has been shown a long time ago that normal hearing listeners can exhibit a high sensitivity to so-called "envelope ITDs" of high frequency carriers (Henning 1974). More recently, Kan et al. (2015) and Litovsky et al. (2012) have also demonstrated ITD sensitivity with electrical stimulation of the basal or middle part of the cochlea in human $\mathrm{Cl}$ listeners. Furthermore, our studies (Buck et al. 2021; Rosskothen-Kuhl et al. 2021) show very high physiological and behavioral sensitivity to ITDs in the rat, even though we target mid frequency regions of the cochlea, regions equivalent to those on the human cochlea that are routinely covered by conventional $\mathrm{Cl}$ electrode arrays. Together, these results strongly suggest that delivering ITDs at any part of the cochlea can be beneficial, and that the difficulty in reaching the most low-frequency parts of the tonotopic array with conventional $\mathrm{Cl}$ electrodes is at best a very a minor limiting factor in the ITD sensitivity that is in principle achievable with bilateral Cls.

\section{Possible causes of better ITD perception in ND biCl rats than in biCl}

\section{human listeners}

Why our rats exhibit much better ITD performance than that previously reported in biCl users is not known, but possible explanations, some of which we have touched on above, include species differences, differences in the nature of the stimulation and/or training effects. Species differences may of course play a role, but a multitude of studies indicate 
365 that rats are a good animal model for human cochlear implantation (Rosskothen-Kuhl and Illing 2010; Rosskothen-Kuhl and Illing 2012; Rosskothen-Kuhl and Illing 2014; Rauch et al. 2016; Rosskothen-Kuhl et al. 2018; Jakob et al. 2019; Rosskothen-Kuhl et al. 2021; Weltin et al. 2022) and binaural hearing (Li et al. 2019; Li et al. 2021; Rosskothen-Kuhl et al. 2021). We thus think species differences are unlikely to be a major factor.

370 As already noted, our setup always provides informative ITDs with microsecond precision from the first stimulation, while clinical processors in current use do not. Devices in clinical use are typically based on two monaural processors with asynchronous clock times and at best modest time locking to the temporal fine structure of the input at each ear. Many human $\mathrm{Cl}$ psychoacoustic studies are performed with research interfaces capable of more 375 precise binaural synchronization, but the vast majority of the auditory experience of the participants nevertheless comes from asynchronous clinical $\mathrm{Cl}$ speech processors which deliver interaural pulse timing patterns that are uninformative and potentially misleading. The differences in ITD sensitivity could simply be driven by the fact that ITDs are a much more useful and reliable cue for our biCl rats than they are for the typical human biCl user.

380 This hypothesis is supported by a recent electrophysiological study by Thompson et al. (2021), which has demonstrated that animals that experienced reliable ITD information through "ITD-aware sound processors" have a higher neuronal ITD sensitivity than biCI animals supplied with standard clinical processors.

It has long been thought that the absence of binaural experience during early life may 385 preclude the development of normal ITD sensitivity in CI listeners (Litovsky 2010; Gordon et al. 2014). However, in Rosskothen-Kuhl et al. (2021) we demonstrate that a long period of hearing deprivation from $\sim$ p15 until early adulthood in our rats ( p70-98) does not impair the ability to use ITDs. In fact, the ITD sensitivity of neonatally deafened, adult implanted rats was found to be comparable to that of normally hearing, acoustically stimulated rats

390 (Li et al. 2019). ITD sensitivity thus does not appear to have a critical period. Providing auditory input with consistently informative ITDs from the onset of stimulation may play a much more important role in the development of ITD sensitivity than ensuring that this initial stimulation happens very early. Unlike typical early deaf human biCl users, our ND $\mathrm{Cl}$ animals were able to lateralize ITDs with thresholds around $50 \mu$ s that appear no worse

395 than those seen in their normally hearing siblings ( $\mathrm{Li}$ et al. 2019), but unlike human $\mathrm{Cl}$ users our animals were also never exposed to prolonged periods of stimulation with arbitrary and uninformative interaural time delays as delivered by clinical speech processors. 
Finally, one needs to consider the possibility that training effects may play a major role.

400 For our biCl rats, lateralizing binaural pulse trains with precise ITDs but no other noteworthy features day after day constituted the entirety of their auditory experience, and they would spend approximately 10 hours a week for several months practicing lateralizing these sounds with instant positive reinforcement for correct performance. In contrast, human $\mathrm{Cl}$ users spend most of their auditory experience trying to make sense of the

405 cacophony of everyday life, and little if any of their time and attention is dedicated to honing their ITD detection skills. In this context it is of interest to note Goldsworthy and Shannon (2014) report that just 32 hours of training could lead to very substantial improvements in $\mathrm{Cl}$ pulse rate discrimination. If monaural temporal processing in human $\mathrm{Cl}$ users can improve with training, then the same may also be true for binaural temporal 410 processing, as rate discrimination and ITD lateralization may share some common physiological mechanisms (Ihlefeld et al. 2015). Generally, psychoacoustic limits observed in largely untrained $\mathrm{Cl}$ listeners may substantially underestimate the physiological limits that the $\mathrm{Cl}$ stimulated auditory pathway is in principle capable of.

Furthermore, recent studies have demonstrated that lateralization training can lead to a

415 reweighting of binaural cues in normal hearing listeners (Kumpik et al. 2019; Klingel et al. 2021) and biCl users (Klingel and Laback 2022), such that the listeners rely more or less heavily on ILDs or ITDs respectively depending on which cue is more reliable or informative. This could be relevant for $\mathrm{Cl}$ listeners, as they could similarly down-weight ITD cues if the ongoing stimulation with clinical processors does not transmit reliable,

420 informative ITDs which would thus encourage a reliance exclusively on ILDs to the detriment of ITDs. Conversely, it is conceivable that targeted ITD lateralization training under optimized bilateral stimulation might allow $\mathrm{Cl}$ listeners to regain and rebalance their sensitivity to ITDs. However, even if training could improve ITD sensitivity in CI listeners for pulse rates up to $900 \mathrm{pps}$, our results in the $\mathrm{Cl}$ animal model provide strong evidence

425 that there is likely to be a physiological upper bound at pulse rates much above 900 pps, given that ITD sensitivity in our rats dropped precipitously at 1800 pps (see Figs. 2, 3, S2, and S3).

\section{Conclusion}

Under synchronized bilateral stimulation, we have shown that, at least in rats, clinical pulse 430 rates of up to 900 pps should not prevent the development of good ITD sensitivity. This is not only important for better sound localization in human $\mathrm{Cl}$ users but also for their speech understanding in noisy environments. Sharp onset stimulus envelopes and lower pulse 
rates can further improve ITD sensitivity, but are not an absolute requirement, as good ITD sensitivity was still observed at 900 pps pulse trains with gentle onset Hanning envelopes.

435 Delivering pulsatile binaural stimulation through neuroprosthetic devices at pulse rates that are fast enough to encode speech envelopes effectively and yet slow enough to allow the precise encoding of timing cues for ITDs may therefore not be as difficult as previously thought. This is an important finding for the development of future $\mathrm{Cl}$ speech processor algorithms which may be able to provide biCl users of the future with a much better 440 binaural listening experience. 


\section{Methods and Materials}

All procedures involving experimental animals reported here were approved by the Department of Health of Hong Kong (\#16-52 to 16-55; 18-81 to 18-83; 20-143 to 20-144 $\mathrm{DH} / \mathrm{HA} \& \mathrm{P} / 8 / 2 / 5)$ and/or the Regierungspräsidium Freiburg (\#35-9185.81/G-17/124), as well as by the appropriate local ethical review committee. Sixteen female Wistar rats were neonatally deafened as described in (Rosskothen-Kuhl and Illing 2012). After verification of hearing loss using acoustic auditory brainstem recordings, young adult rats (postnatal weeks 10-14) were implanted simultanously with bilateral Cls from PEIRA (animal arrays

450 ST08.45, ST05.45 or ST03.45, Cochlear Ltd, Peira, Beerse, Belgium) or with bilateral Cls kindly provided by MED-EL (3-patch animal arrays, Medical Electronics, Innsbruck, Austria). All rats underwent behavioral training as described in Rosskothen-Kuhl et al. (2021) (Fig. 1). As before, animals received binaurally synchronized input from the first stimulation. For details on the electric stimuli and stimulation setup see Rosskothen-Kuhl 455 et al. (2021).

\section{Psychoacoustic Training and Testing}

The ability to discriminate ITDs of $\mathrm{Cl}$ stimuli was tested in a 2-AFC ITD lateralization task in our custom made behavior setup as described in Rosskothen-Kuhl et al. (2021).

460 Training and testing for the 16 animals was done in a pseudo random order following implantation (Fig. 1). Animals were tested on four pulse rates, 50, 300, 900, and 1800 pps, with rectangle $(\mathrm{N}=16)$ and Hanning $(\mathrm{N}=10)$ windowed pulse trains. For this experiment, both individual pulses and envelopes carried the same ITD information by keeping the fine structure and envelope ITDs equal for all trials. As in Rosskothen-Kuhl et al. (2021), the

465 ITDs were presented between -150 and $+150 \mu \mathrm{s}$, where negative represents a left leading and positive a right leading ITD. This range covers $125 \%$ of the animal's physiological range, which is between -120 and $+120 \mu$ s (Koka et al. 2008). Each pulse train had a duration of $200 \mathrm{~ms}$, and animals were stimulated at a maximum of $6 \mathrm{~dB}(0 \mathrm{~dB}$ relative to $100 \mu \mathrm{A}$ peak pulse amplitude) above their eABR thresholds (see Rosskothen-Kuhl et al.

470 (2021) for details). The eABRs of an example rat are shown in Figure S1 directly after implantation, 6 weeks, and 6 months post implantation. The starting frequency of the first training was assigned pseudo randomly to rule out any effects from the order in which they were trained and tested. Hanning windowed pulse trains consisted of a raised cosine waveform with a $100 \mathrm{~ms}$ rising and falling phase, respectively (Fig. 5). 
If animals struggled with the higher frequencies (particularly $1800 \mathrm{pps}$ ) in training sessions, these rates were interleaved with trials at easier pulse rates in order to keep the animals motivated and to allow them to obtain sufficient water rewards for stimuli with both rectangular and Hanning windowed envelopes. All $16 \mathrm{Cl}$ animals were tested for ITD sensitivity under rectangular windowed stimuli at 300 and 900 pps. For rectangular windowed stimuli at pulse rates of 50 and 1800 pps, 14 and $8 \mathrm{Cl}$ animals were tested, respectively (Figs. 1, 2, S2). For ITD sensitivity under Hanning windowed envelope, ten $\mathrm{Cl}$ animals were tested at 50,300, and 900 pps, and six of them were additionally tested at 1800 pps (Figs. 1, 3, S3).
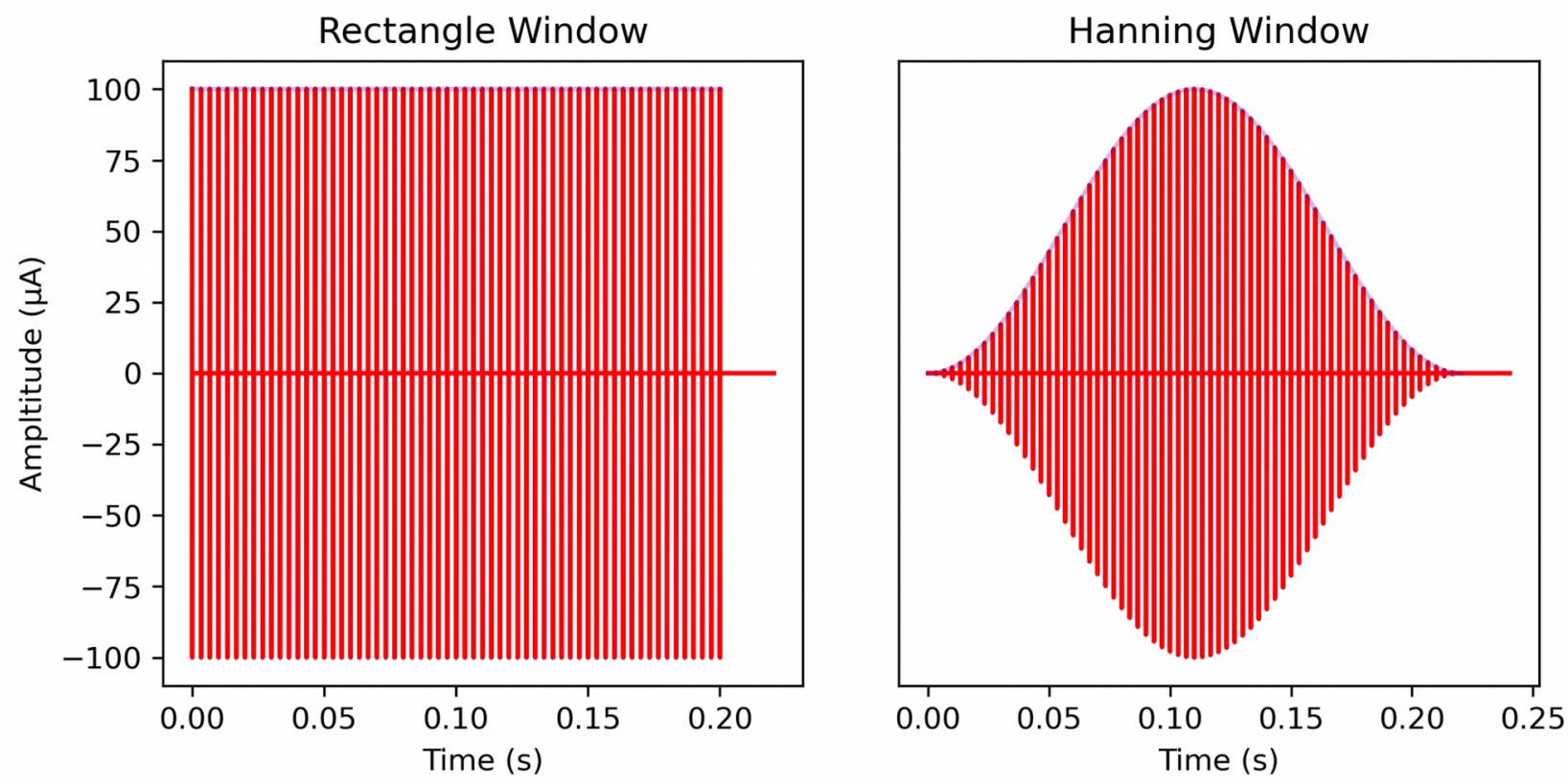

Figure 5: Waveforms of the electrical stimuli for rectangular (left) and Hanning (right) windowed stimulation. Waveforms shown are examples at $300 \mathrm{pps}$.

\section{Data Analysis}

Psychometric curve fits were estimated as described in Rosskothen-Kuhl et al. (2021). To determine behavioral ITD sensitivity, the proportions of "right" responses an animal made as a function of stimulus ITD were fitted using either linear or cumulative Gaussian sigmoid functions (Figs. 2, 3). Whether the sigmoid model gave a better fit than the linear model for a given animal and a given combination of stimulus envelope and pulse rate was determined using a deviance test. Once the best fit model was chosen, the slope of the fitted psychometric curve at ITD=0 was computed in units of $\%$ change in the animal's preference for choosing the right water spout per microsecond increase in the value of 
495 stimulus ITD. This slope served as a metric for the behavioral sensitivity of the animal to ITD at the given stimulus envelope and pulse rate.

In order to be able to estimate confidence limits and perform statistical inferences on these psychometric slope ITD sensitivity metrics, we then performed a bootstrap resampling analysis. The first step of this analysis consisted of an $\mathrm{N}$-out-of- $\mathrm{N}$ resampling of the

500 behavioral data to obtain bootstrap distributions of slope values. For each animal, we collected the $\mathrm{N}$ trials the animal performed for a given combination of ITD values, envelope and pulse rate, and drew, with replacement, a bootstrap sample of $\mathrm{N}$ trials from that set. Readers versed in probability theory may recognize that this step is equivalent to drawing a random number from a binomial distribution with a "hit" probability equal to the fraction of 505 "right" choices made by the animal for this particular stimulus. After collecting these resampled trials for each ITD tested at the given envelope and pulse rate, the slope of the psychometric function for the resampled data was computed. Repeating this process 1000 times yielded a set of bootstrapped psychometric slopes. These were computed in turn for each animal, envelope type and stimulus pulse rate, and could serve to compute

510 confidence intervals or as empirical distributions which could be further resampled for statistical inference on the effects of changes in stimulus pulse rate or envelope.

To examine the statistical significance of effects in envelope type or pulse rate changes, we decided to carry out 16 pairwise comparisons in total: 6 comparisons for each pair of pulse rates with rectangular envelopes (see Tab. 1), 6 with Hanning envelopes (see Tab.

515 2), and 4 comparing the two envelope types at each pulse rate tested (see Tab. 3). Because it was not possible to test all animals on every stimulus parameter combination, and because we wished to keep our statistical analysis "within subject", in order to decide whether changing envelope type or pulse rates had a significant effect, we first identified the subset of animals which were tested at both of the stimulus parameter combinations to

520 be compared. We then carried out resampling of the previously calculated bootstrapped psychometric slopes for each animal to generate mean bootstrap slopes for this cohort. For each animal, one bootstrap slope value was drawn for each of the two stimulus types to be compared, and the values were averaged across the cohort to produce a pair of mean slopes. This process was repeated 16,000 times to generate 16,000 pairs of cohort 525 averaged bootstrap slopes $\left[\mathrm{s}_{1}, \mathrm{~s}_{2}\right]_{\mathrm{N}}, \mathrm{N} \in\{1 \ldots 16,000\}$. An $\mathrm{N}$ of 16,000 repeats was chosen to resolve a smallest $p$-value of 0.001 after multiple comparison correction (see below). These pairs of resampled, cohort-averaged psychometric slopes were then used to calculate the $p$-value of the null hypothesis that there were no significant differences 
between the values $s_{1}, s_{2}$ in each pair on average. We first determined whether $s_{1}$ or $s_{2}$

530 had the smaller mean when averaged over the $N$ cohort resampling trials. Let $S_{S}$ and $S_{L}$ denote the values in each pair that had the smaller $(S)$ or larger $(L)$ mean across $N$, respectively. Finally, we computed the proportion of the 16,000 pairs for which $S_{S}>S_{L}$. If there was no significant difference in the slopes for each condition, then we would expect $\mathrm{S}_{\mathrm{S}} \approx \mathrm{S}_{\mathrm{L}}$, and $\mathrm{S}_{\mathrm{S}}>\mathrm{S}_{\llcorner}$should then be true almost half the time, but if there was a significant

535 difference, $\mathrm{S}_{\mathrm{S}}>\mathrm{S}_{\mathrm{L}}$ should be observed only rarely. The proportion of cases for which $\mathrm{S}_{\mathrm{S}}>\mathrm{S}_{\mathrm{L}}$ can therefore serve as an uncorrected p-value for the null hypothesis that the psychometric slopes for each of the two conditions under consideration did not differ significantly. This uncorrected p-value was then multiplied by 16 , the total number of comparisons to be performed, and if necessary limited to a maximum of 1 , to generate the

$540 \mathrm{p}$-values reported in Tables 1-3. After multiple comparison correction, the smallest $p$-value that this resampling method can resolve is 0.001 , and $p$-values of 0 returned by this method are therefore reported as $<0.001$.

The estimated 75\% ITD discrimination thresholds were determined from the slopes exhibited by the fitted psychometric curves at $0 \mu$ ITD. As can be seen by inspection of

545 Figures 2, 3, S2, and S3, the psychometrics were either fit directly with a straight-line equation (linear model) or were reasonably well approximated by a straight line tangent over the range of $25-75 \%$ right responses (green dashed lines on the sigmoid psychometric functions) with the slope just mentioned. In other words, the psychometric can be reasonably well approximated with a straight line of a constant slope over the

550 range of $25-75 \%$ right responses. Consequently, one can estimate the $75 \%$ discrimination threshold easily as the change in ITD that would be required to "climb" along the (tangent to the) psychometric function by $25 \%$ points, from the $50 \%$ level of completely random guessing to the level of $75 \%$ correct "right" responses. Note that, due to symmetry, the same absolute change in ITD is required whether one climbs or descends by $25 \%$ points

555 along the tangent line. Let $s$ denote the slope of the (tangent to the) psychometric in $\% / \mu$, and $\theta_{75}$ denote the $75 \%$ discrimination threshold in $\mu \mathrm{s}$, then, as just explained, $s \cdot \theta_{75}=25$, or equivalently, $\theta_{75}=25 / \mathrm{s}$. The mean of the $75 \%$ ITD discrimination thresholds, estimated using this simple formula and averaged across animals, are reported in Table 4 for each of the eight different stimulation conditions tested. These $75 \%$ thresholds may slightly 560 underestimate thresholds that might be observed in ITD discrimination tasks. First, because they ignore the (usually small) effect of non-linearity for sigmoidal psychometrics, and second, because they estimate the threshold around the animal's subjective $50 \%$ ITD value, rather than at ITD $=0$. This offsets the possible effects of a small ear-bias which 
might elevate thresholds in other types of discrimination tasks, depending on the task

565 details. Readers who are interested in precise comparisons of ITD thresholds across studies and species need to keep methodological differences in mind. 


\section{Supplementary Materials:}
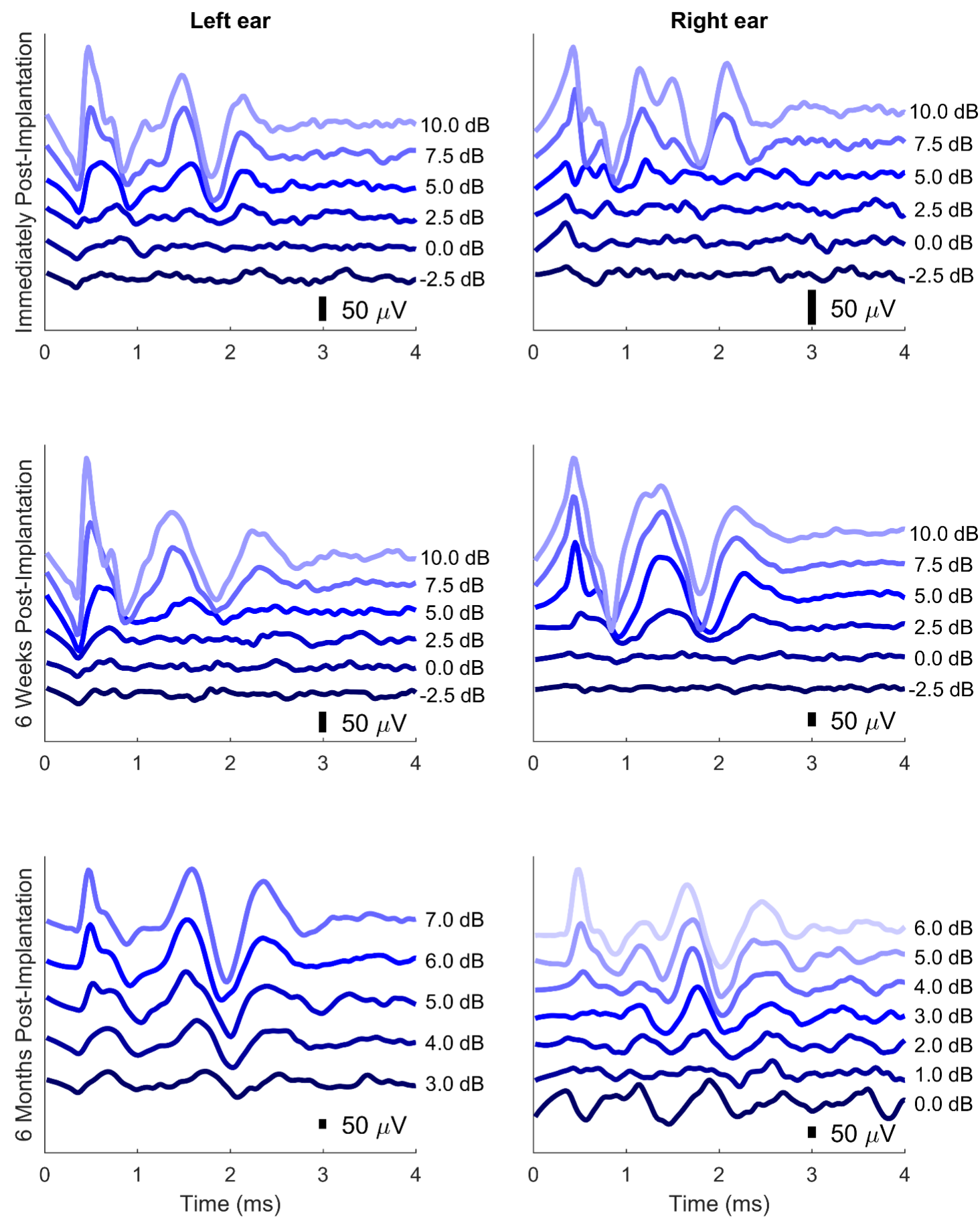

Figure S1: Example eABRs showing immediately after bilateral $\mathrm{Cl}$ implantation, 6 weeks, and 6 months after implantation thresholds for the left and right ears of a neonatally deafened rat. Scale bars are shown in each plot with reference to $50 \mu \mathrm{V}$. Colour represents a different SPL with dark to light colors going from softest to loudest, and $0 \mathrm{~dB}$ SPL corresponding to $100 \mu \mathrm{A}$ current level. Electric artefacts have been removed using interpolation over the duration of the stimulus. For details on the stimulus and presentation, see Rosskothen-Kuhl et al. (2021). 

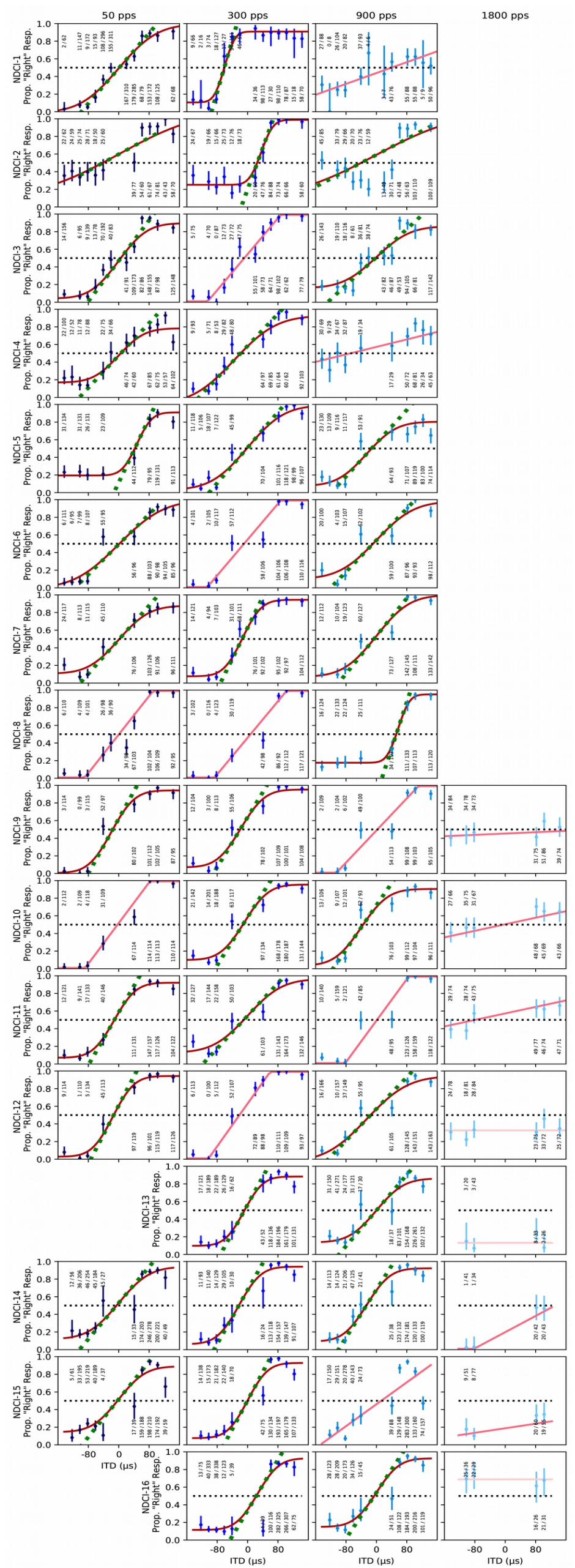
Figure S2: Psychometric functions of all $16 \mathrm{Cl}$ rats for rectangular windowed pulse trains at each pulse rate. Each column represents a different pulse rate from 50, 300, 900 and 1800 pulses per second (pps) (left to right). Each row shows the responses for a given animal. The y-axis reflects "right" responses where "right" refers to the right hand spout (Prop. "Right" Resp.). The x-axis shows the tested interaural time difference (ITD) values from -150 to $+150 \mu \mathrm{s}$. Negative ITD values indicate left leading ITDs. Annotations above or below each marker indicate the number of trials the animal chose the right hand side spout over the total number of presentations for a given ITD value. From dark to light the different shades of red indicate sigmoid, linear with bounds or null model psychometric curve fits. Green dashed lines show slopes of psychometric curves at ITD=0. Slopes serve to quantify the behavioral sensitivity of the animal to ITD. 

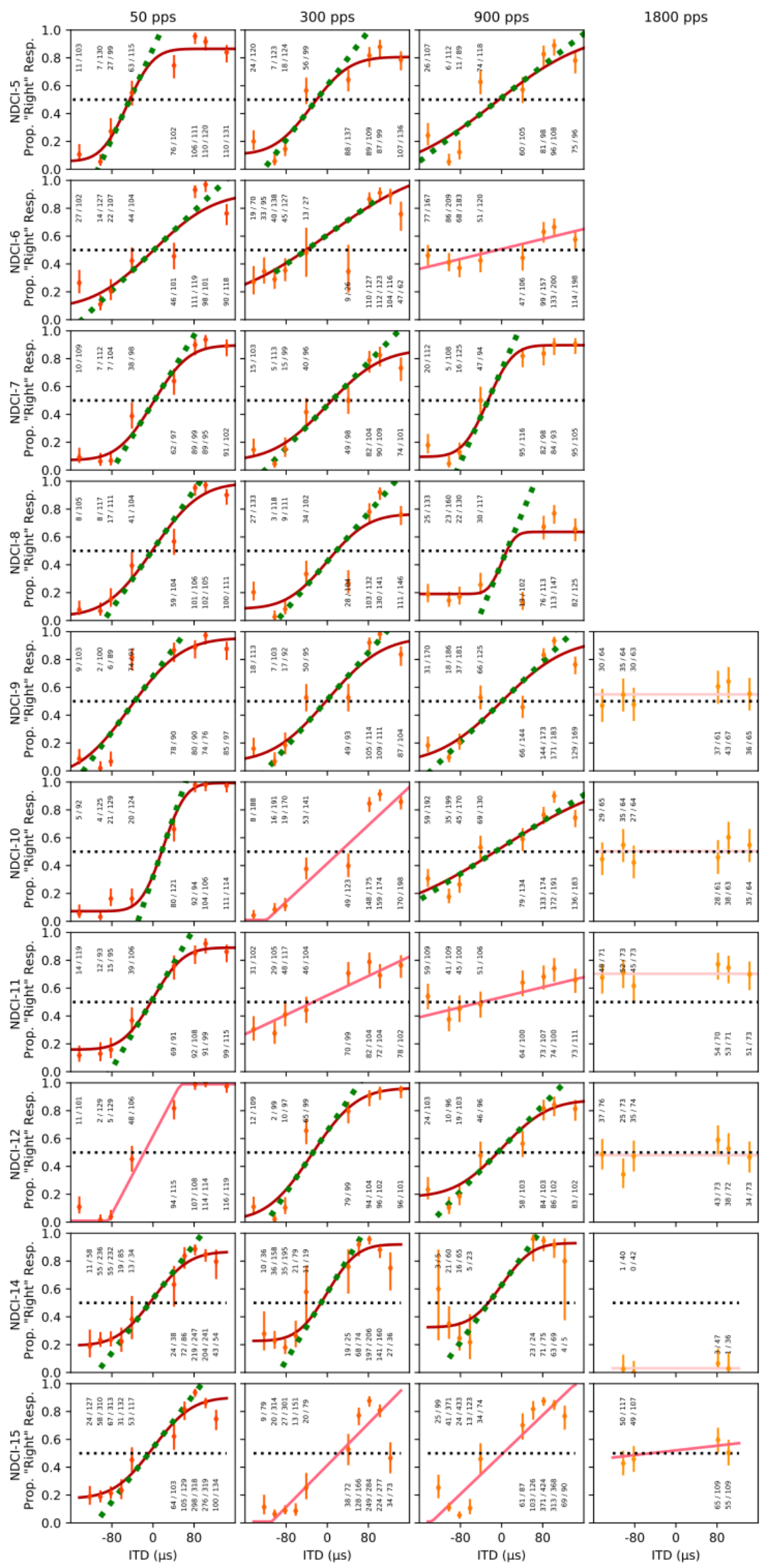

Figure S3: Psychometrics of $10 \mathrm{Cl}$ rats for Hanning windowed pulse trains with varying ITDs as in Figure S2. Details are as for S2. 


\section{Acknowledgements}

We thank Felix Kleinschroth for his assistance in figure design as well as Felix

575 Kleinschroth, Stella Mayer, and Lakshay Khurana for assisting behavioral training of $\mathrm{Cl}$ rats. Work leading to this publication was supported by grants from the Hong Kong General Research Fund (11100219 and 11101020), the Shenzhen Science and Innovation Fund Grant (JCYJ20180307124024360), Health and Medical Research Fund (07181406), the German Academic Exchange Service (DAAD) with funds from the German Federal

580 Ministry of Education and Research (BMBF) and the People Programme (Marie Curie Actions) of the European Union's Seventh Framework Programme (FP7/2007-2013) under REA grant agreement $n^{\circ} 605728$ (PRIME - Postdoctoral Researchers International Mobility Experience), the Research Commission of the Medical Faculty of the Medical Center - University of Freiburg, and friends' association “Taube Kinder lernen hören e. V.“. 585 Eight cochlear implant animal arrays were kindly provided by MED-EL Medical Electronics, Innsbruck, Austria (Research Agreement PVFR2019/2).

\section{Declaration of Interests}

The authors declare no competing interests. 


\section{References}

Brown, A. D. and Stecker, G. C. (2010) Temporal weighting of interaural time and level differences in high-rate click trains. J Acoust Soc Am 128:332-341.

595 Buck, A. N., Rosskothen-Kuhl, N. and Schnupp, J. W. (2021) Sensitivity to interaural time differences in the inferior colliculus of cochlear implanted rats with or without hearing experience.. Hearing research 408:108305.

Buechel, B. D., Hancock, K. E., Chung, Y. and Delgutte, B. (2018) Improved Neural Coding of ITD with Bilateral Cochlear Implants by Introducing Short Inter-pulse Intervals.. Journal of the Association for Research in Otolaryngology : JARO 19:681-702.

Chung, Y., Buechel, B. D., Sunwoo, W., Wagner, J. D. and Delgutte, B. (2019) Neural ITD Sensitivity and Temporal Coding with Cochlear Implants in an Animal Model of EarlyOnset Deafness.. Journal of the Association for Research in Otolaryngology : JARO 20:3756.

605 Chung, Y., Hancock, K. E. and Delgutte, B. (2016) Neural Coding of Interaural Time Differences with Bilateral Cochlear Implants in Unanesthetized Rabbits. J Neurosci 36:5520-5531.

Chung, Y., Hancock, K. E., Nam, S.-I. and Delgutte, B. (2014) Coding of Electric Pulse Trains Presented through Cochlear Implants in the Auditory Midbrain of Awake Rabbit: Comparison with Anesthetized Preparations. J Neurosci 34:218-231.

Conti-Ramsden, G., St Clair, M. C., Pickles, A. and Durkin, K. (2012) Developmental trajectories of verbal and nonverbal skills in individuals with a history of specific language impairment: from childhood to adolescence.. J. Speech. Lang. Hear. Rees 55:1716-1735.

Ehlers, E., Goupell, M. J., Zheng, Y., Godar, S. P. and Litovsky, R. Y. (2017) Binaural sensitivity in children who use bilateral cochlear implants. J Acoust Soc Am 141:42644277.

Eklöf, M. and Tideholm, B. (2018) The choice of stimulation strategy affects the ability to detect pure tone inter-aural time differences in children with early bilateral cochlear implantation.. Acta oto-laryngologica 138:554-561.

620 Goldsworthy, R. L. and Shannon, R. V. (2014) Training improves cochlear implant rate discrimination on a psychophysical task.. The Journal of the Acoustical Society of America 135:334-341.

Gordon, K. A., Deighton, M. R., Abbasalipour, P. and Papsin, B. C. (2014) Perception of binaural cues develops in children who are deaf through bilateral cochlear implantation.

625 PLoS One 9:e114841.

Goupell, M. J. and Litovsky, R. Y. (2014) The effect of interaural fluctuation rate on correlation change discrimination. JARO 15:115-129.

Grantham, D. W., Ashmead, D. H., Ricketts, T. A., Haynes, D. S. and Labadie, R. F. (2008) Interaural time and level difference thresholds for acoustically presented signals in post-lingually deafened adults fitted with bilateral cochlear implants using CIS+ 
processing.. Ear and hearing 29:33-44.

Green, T., Faulkner, A. and Rosen, S. (2012) Variations in carrier pulse rate and the perception of amplitude modulation in cochlear implant users.. Ear and hearing 33:221230.

635 Hancock, K. E., Chung, Y. and Delgutte, B. (2012) Neural ITD coding with bilateral cochlear implants: effect of binaurally coherent jitter. J Neurophysiol 108:714-728.

Hancock, K. E., Chung, Y. and Delgutte, B. (2013) Congenital and prolonged adult-onset deafness cause distinct degradations in neural ITD coding with bilateral cochlear implants. JARO 14:393-411.

640 Haqqee, Z., Valdizón-Rodríguez, R. and Faure, P. A. (2021) High frequency sensitivity to interaural onset time differences in the bat inferior colliculus.. Hearing research 400:108133.

Henning, G. B. (1974) Detectability of interaural delay in high-frequency complex waveforms.. The Journal of the Acoustical Society of America 55:84-90.

645 van Hoesel, R., Ramsden, R. and Odriscoll, M. (2002) Sound-direction identification, interaural time delay discrimination, and speech intelligibility advantages in noise for a bilateral cochlear implant user.. Ear and hearing 23:137-149.

van Hoesel, R. J. (2008) Observer weighting of level and timing cues in bilateral cochlear implant users. J Acoust Soc Am 124:3861-3872.

650 van Hoesel, R. J. and Clark, G. M. (1997) Psychophysical studies with two binaural cochlear implant subjects.. The Journal of the Acoustical Society of America 102:495-507. van Hoesel, R. J. M. (2004) Exploring the Benefits of Bilateral Cochlear Implants. Audiol Neurootol 9:234-246.

van Hoesel, R. J. M. (2007) Sensitivity to binaural timing in bilateral cochlear implant users. J Acoust Soc Am 121:2192-2206.

van Hoesel, R. J. M., Jones, G. L. and Litovsky, R. Y. (2009) Interaural time-delay sensitivity in bilateral cochlear implant users: effects of pulse rate, modulation rate, and place of stimulation. JARO 10:557-567.

van Hoesel, R. J. M. and Tyler, R. S. (2003) Speech perception, localization, and 660 lateralization with bilateral cochlear implants.. The Journal of the Acoustical Society of America 113:1617-1630.

Ihlefeld, A., Carlyon, R. P., Kan, A., Churchill, T. H. and Litovsky, R. Y. (2015) Limitations on Monaural and Binaural Temporal Processing in Bilateral Cochlear Implant Listeners.. Journal of the Association for Research in Otolaryngology : JARO 16:641-652.

665 Jakob, T. F., Illing, R.-B. and Rosskothen-Kuhl, N. (2019) Monaural Neonatal Deafness Induces Inhibition among Bilateral Auditory Networks under Binaural Activation.. Neuroscience 400:1-16.

Joris, P. X. and Yin, T. C. (1998) Envelope coding in the lateral superior olive. III. Comparison with afferent pathways. J Neurophysiol 79:253-269. 
670 Kan, A., Jones, H. G. and Litovsky, R. Y. (2015) Effect of multi-electrode configuration on sensitivity to interaural timing differences in bilateral cochlear-implant users. J Acoust Soc Am 138:3826-3833.

Kan, A. and Litovsky, R. Y. (2015) Binaural hearing with electrical stimulation. Hear Res 322:127-137.

675 Klingel, M., Kopčo, N. and Laback, B. (2021) Reweighting of Binaural Localization Cues Induced by Lateralization Training.. Journal of the Association for Research in Otolaryngology : JARO 22:551-566.

Klingel, M. and Laback, B. (2022) Reweighting of Binaural Localization Cues in Bilateral Cochlear-Implant Listeners.. Journal of the Association for Research in Otolaryngology : 680 JARO 23:119-136.

Koka, K., Read, H. L. and Tollin, D. J. (2008) The acoustical cues to sound location in the rat: measurements of directional transfer functions. J Acoust Soc Am 123:4297-4309.

Kral, A. (2013) Auditory critical periods: a review from system's perspective.. Neuroscience 247:117-133.

685 Kumpik, D. P., Campbell, C., Schnupp, J. W. H. and King, A. J. (2019) Re-weighting of Sound Localization Cues by Audiovisual Training.. Frontiers in neuroscience 13:1164.

Laback, B., Egger, K. and Majdak, P. (2015) Perception and coding of interaural time differences with bilateral cochlear implants. Hear Res 322:138-150.

Laback, B., Majdak, P. and Baumgartner, W.-D. (2007) Lateralization discrimination of 690 interaural time delays in four-pulse sequences in electric and acoustic hearing. J Acoust Soc Am 121:2182-2191.

Laback, B., Pok, S.-M., Baumgartner, W.-D., Deutsch, W. A. and Schmid, K. (2004) Sensitivity to interaural level and envelope time differences of two bilateral cochlear implant listeners using clinical sound processors.. Ear and hearing 25:488-500.

695 Laback, B., Zimmermann, I., Majdak, P., Baumgartner, W.-D. and Pok, S.-M. (2011) Effects of envelope shape on interaural envelope delay sensitivity in acoustic and electric hearing. J Acoust Soc Am 130:1515-1529.

Lapid, E., Ulrich, R. and Rammsayer, T. (2008) On estimating the difference limen in duration discrimination tasks: a comparison of the 2AFC and the reminder task..

700 Perception \& psychophysics 70:291-305.

Li, K., Auksztulewicz, R., Chan, C. H. K., Mishra, A. P. and Schnupp, J. W. H. (2021) The precedence effect in spatial hearing emerges only late in the auditory pathway, .

Li, K., Chan, C. H. K., Rajendran, V. G., Meng, Q., Rosskothen-Kuhl, N. and Schnupp, J. W. H. (2019) Microsecond sensitivity to envelope interaural time differences in rats.. The 705 Journal of the Acoustical Society of America 145:EL341.

Litovsky, R. (2010) Bilateral Cochlear Implants. ASHA Leader 15:14.

Litovsky, R. Y., Ehlers, E., Hess, C. and Harris, S. (2013) Reaching for sound measures: an ecologically valid estimate of spatial hearing in 2- to 3-year-old children with bilateral 
cochlear implants.. Otology \& neurotology : official publication of the American Otological

710 Society, American Neurotology Society [and] European Academy of Otology and Neurotology 34:429-435.

Litovsky, R. Y. and Gordon, K. (2016) Bilateral cochlear implants in children: Effects of auditory experience and deprivation on auditory perception. Hear Res 338:76-87.

Litovsky, R. Y., Goupell, M. J., Godar, S., Grieco-Calub, T., Jones, G. L., Garadat, S. N.,

715 Agrawal, S., Kan, A., Todd, A., Hess, C. and others (2012) Studies on bilateral cochlear implants at the University of Wisconsin's Binaural Hearing and Speech Laboratory. Journal of the American Academy of Audiology 23:476-494.

Majdak, P., Laback, B. and Baumgartner, W.-D. (2006) Effects of interaural time differences in fine structure and envelope on lateral discrimination in electric hearing. $J$

720 Acoust Soc Am 120:2190-2201.

Middlebrooks, J. C. (2008) Cochlear-implant high pulse rate and narrow electrode configuration impair transmission of temporal information to the auditory cortex.. Journal of neurophysiology 100:92-107.

Middlebrooks, J. C. and Snyder, R. L. (2007) Auditory prosthesis with a penetrating nerve

725 array.. Journal of the Association for Research in Otolaryngology : JARO 8:258-279.

Middlebrooks, J. C. and Snyder, R. L. (2010) Selective electrical stimulation of the auditory nerve activates a pathway specialized for high temporal acuity.. The Journal of neuroscience : the official journal of the Society for Neuroscience 30:1937-1946.

Noel, V. A. and Eddington, D. K. (2013) Sensitivity of bilateral cochlear implant users to

730 fine-structure and envelope interaural time differences.. The Journal of the Acoustical Society of America 133:2314-2328.

Poon, B. B., Eddington, D. K., Noel, V. and Colburn, H. S. (2009) Sensitivity to interaural time difference with bilateral cochlear implants: Development over time and effect of interaural electrode spacing. J Acoust Soc Am 126:806-815.

735 Rauch, A.-K., Rosskothen-Kuhl, N. and Illing, R.-B. (2016) Counter-regulation of the AP-1 monomers pATF2 and Fos: Molecular readjustment of brainstem neurons in hearing and deaf adult rats after electrical intracochlear stimulation. Neuroscience 313:184-198.

Rosskothen-Kuhl, N., Buck, A. N., Li, K. and Schnupp, J. W. (2021) Microsecond interaural time difference discrimination restored by cochlear implants after neonatal deafness.. eLife 74010.

Rosskothen-Kuhl, N., Hildebrandt, H., Birkenhäger, R. and Illing, R.-B. (2018) Astrocyte Hypertrophy and Microglia Activation in the Rat Auditory Midbrain Is Induced by Electrical Intracochlear Stimulation. Front Cell Neurosci 12.

Rosskothen-Kuhl, N. and Illing, R.-B. (2010) Nonlinear development of the populations of 745 neurons expressing c-Fos under sustained electrical intracochlear stimulation in the rat auditory brainstem. Brain Res 1347:33-41.

Rosskothen-Kuhl, N. and Illing, R.-B. (2012) The impact of hearing experience on signal integration in the auditory brainstem: A c-Fos study of the rat. Brain Res 1435:40-55. 
Rosskothen-Kuhl, N. and Illing, R.-B. (2014) Gap43 transcription modulation in the adult brain depends on sensory activity and synaptic cooperation. PLoS One 9:e92624.

Schnupp, J., Nelken, I. and King, A., 2011 Auditory neuroscience : making sense of sound. MIT Press.

Shannon, R. V., Cruz, R. J. and Galvin, J. J. (2011) Effect of stimulation rate on cochlear implant users' phoneme, word and sentence recognition in quiet and in noise.. Audiology \& neuro-otology 16:113-123.

Smith, Z. M. and Delgutte, B. (2007) Sensitivity to interaural time differences in the inferior colliculus with bilateral cochlear implants. J Neurosci 27:6740-6750.

Snyder, R., Leake, P., Rebscher, S. and Beitel, R. (1995) Temporal resolution of neurons in cat inferior colliculus to intracochlear electrical stimulation: effects of neonatal deafening and chronic stimulation.. Journal of neurophysiology 73:449-467.

Srinivasan, S., Laback, B., Majdak, P. and Delgutte, B. (2018) Introducing Short Interpulse Intervals in High-Rate Pulse Trains Enhances Binaural Timing Sensitivity in Electric Hearing. JARO 19:301-315.

Stakhovskaya, O., Sridhar, D., Bonham, B. H. and Leake, P. A. (2007) Frequency map for 765 the human cochlear spiral ganglion: implications for cochlear implants.. Journal of the Association for Research in Otolaryngology : JARO 8:220-233.

Sunwoo, W., Delgutte, B. and Chung, Y. (2021) Chronic Bilateral Cochlear Implant Stimulation Partially Restores Neural Binaural Sensitivity in Neonatally-Deaf Rabbits.. The Journal of neuroscience : the official journal of the Society for Neuroscience 41:3651-3664.

770 Thakkar, T., Anderson, S. R., Kan, A. and Litovsky, R. Y. (2020) Evaluating the Impact of Age, Acoustic Exposure, and Electrical Stimulation on Binaural Sensitivity in Adult Bilateral Cochlear Implant Patients.. Brain sciences 10.

Thakkar, T., Kan, A., Jones, H. G. and Litovsky, R. Y. (2018) Mixed stimulation rates to improve sensitivity of interaural timing differences in bilateral cochlear implant listeners. $J$

775 Acoust Soc Am 143:1428.

Thompson, A. C., Irvine, D. R. F. and Fallon, J. B. (2021) Provision of interaural time difference information in chronic intracochlear electrical stimulation enhances neural sensitivity to these differences in neonatally deafened cats.. Hearing research 406:108253.

Tillein, J., Hubka, P. and Kral, A. (2016) Monaural Congenital Deafness Affects Aural

780 Dominance and Degrades Binaural Processing.. Cereb Cortex 26:1762-1777.

Tillein, J., Hubka, P., Syed, E., Hartmann, R., Engel, A. and Kral, A. (2009) Cortical representation of interaural time difference in congenital deafness. Cereb Cortex 20:492506.

Ulrich, R. and Vorberg, D. (2009) Estimating the difference limen in 2AFC tasks: pitfalls 785 and improved estimators.. Attention, perception \& psychophysics 71:1219-1227.

Vollmer, M., Snyder, R. L., Leake, P. A., Beitel, R. E., Moore, C. M. and Rebscher, S. J. (1999) Temporal properties of chronic cochlear electrical stimulation determine temporal 
resolution of neurons in cat inferior colliculus.. Journal of neurophysiology 82:2883-2902. Weltin, A., Kieninger, J., Urban, G. A., Buchholz, S., Arndt, S. and Rosskothen-Kuhl, N. 790 (2022) Standard cochlear implants as electrochemical sensors: Intracochlear oxygen measurements in vivo.. Biosensors \& bioelectronics 199:113859. 